\title{
On Polynomials with Coefficients of Modulus One
}

\section{Journal Article}

Author(s):

Byrnes, J. S.

Publication date:

1977

Permanent link:

https://doi.org/10.3929/ethz-b-000422579

Rights / license:

In Copyright - Non-Commercial Use Permitted

Originally published in:

Bulletin of the London Mathematical Society 9(2), https://doi.org/10.1112/blms/9.2.171 


\section{ON POLYNOMIALS WITH COEFFICIENTS OF MODULUS ONE}

\section{J. S. BYRNES}

\section{Introduction}

As in Littlewood [5], we let $\mathscr{G}_{n}$ be the class of all polynomials of the form

$$
g_{n}(\theta)=\sum_{k=0}^{n} \exp \left(\alpha_{k} i\right) z^{k}
$$

where the $\alpha_{k}$ are arbitrary real constants and $z=\exp (2 \pi i \theta)$. Clearly $\left\|g_{n}\right\|_{L^{2}}=(n+1)^{\frac{1}{t}}$ for all $g_{n} \in \mathscr{G}_{n}$, and the question "how close can such a $g_{n}$ come to satisfying $\left|g_{n}\right| \equiv(n+1)^{ \pm}$?" has long been the object of intense study. In [5] Littlewood conjectured that there are positive absolute constants $A_{1}$ and $A_{2}$ such that, for arbitrarily large $n$, there exist $g_{n} \in \mathscr{G}_{n}$ with $A_{1} n^{\frac{1}{2}} \leqslant\left|g_{n}(\theta)\right| \leqslant A_{2} n^{\frac{1}{2}}$ for all 0 . In [3] Erdös conjectured that there is a universal constant $c>0$ such that for $n \geqslant 2$, $\left\|g_{n}\right\|_{\infty} \geqslant(1+c) n^{\frac{1}{2}}$ for all $g_{n} \in \mathscr{G}_{n}$.

It was shown by Littlewood [5] that the function

$$
g(\theta)=\sum_{m=0}^{n} \exp \left(\frac{1}{2} m(m+1) \pi i(n+1)^{-1}\right) z^{m}
$$

satisfies: (i) for any $\delta>0,|g| n^{-\frac{1}{2}} \rightarrow 1$ uniformly in $n^{-\frac{1}{2}+\delta} \leqslant|\theta| \leqslant \frac{1}{2}$, and (ii) $|g| \leqslant 1 \cdot 4 n^{\frac{1}{2}}$ for all $\theta$. In the first part of this paper we strengthen part (i) of the Littlewood result in two ways by producing polynomials $g$ which yield an improved estimate for $\left.|g|\right|^{-\frac{1}{2}}$ in a larger subset of the unit circle. In the second part we use the methods already developed to construct functions which are "almost" in $\mathscr{G}_{n}$ and which satisfy the Littlewood conjecture with (within the error) $A_{1}=A_{2}=1$.

1. To begin our work, we require two elementary lemmas.

LEMMA 1. Let

$$
F(x, T)=\left(1-e^{2 \pi i x}\right) \sum_{m=0}^{\infty} \frac{e^{2 \pi i m x}}{m+T},
$$

where $T>1$ and $F$ is defined by continuity when $x$ is an integer. Then $|F(x, T)|<3 / T$ for all $x$.

Proof of Lemma 1. Let $x$ be fixed and not an integer. Then

$$
F(x, T)=\lim _{R \rightarrow \infty}\left(1-e^{2 \pi i x}\right) \sum_{m=0}^{R} \frac{e^{2 \pi i m x}}{m+T}=\frac{1}{T}-\frac{1}{T} \sum_{m=1}^{\infty} \frac{e^{2 \pi i m x}}{\left(m^{2}-m\right) T^{-1}+2 m+T-1},
$$

Received 4 September, 1975; revised 20 May, 1976.

[BULl. LoNDON MATH. Soc., 9 (1977), 171-176] 
and

$$
\sum_{m=1}^{\infty} \frac{1}{\left(m^{2}-m\right) T^{-1}+2 m+T-1}=\sum_{1}^{[T]}+\sum_{[T]+1}^{\infty}<1+T \int_{T}^{\infty} \frac{d x}{x^{2}}=2,
$$

completing the proof of Lemma 1.

LEMMA 2. If $r$ and $x$ are not integers, then

$$
\sum_{m=-\infty}^{\infty} \frac{e^{2 \pi i m x}}{m+r}=\frac{2 \pi i e^{2 \pi i k r}}{1-e^{-2 \pi i r}} e^{-2 \pi i r x}
$$

where $k=[x]$.

Proof of Lemma 2. Simply compute the Fourier Series of the function $F(x)$ of period 1 which, for $0 \leqslant x<1$, is given by

$$
F(x)=\frac{2 \pi i}{1-e^{-2 \pi \mathrm{ir}}} e^{-2 \pi i r x} .
$$

Employing these Lemmas, we are able to prove our basic result.

THEOREM 1. Let $N$ be a positive integer, and define the function

$$
P \in \mathscr{G}_{N^{2}-1} \text { by } P(\theta)=\sum_{k=0}^{N-1} \sum_{j=0}^{N-1} \exp \left(2 \pi i j k N^{-1}\right) z^{j+k N}, \quad z=\exp (2 \pi i \theta) .
$$

Then

(a) $\left|P\left(\frac{j}{N^{2}}\right)\right|=N$ for all integers $j$,

(b) For any $\varepsilon, N^{-1}<\varepsilon<\frac{1}{2}, \quad|P(\theta)|=N+E$ for $-1+\varepsilon \leqslant \theta \leqslant-\varepsilon$, where $|E|<1+2 \pi^{-1}+5(\pi \varepsilon)^{-1}$,

(c) For $N$ odd, $P\left(\frac{1}{2 N}\right)=O(1)$, while for $N$ even, $P\left(\frac{N-1}{2 N^{2}}\right)=O(1)$, and

(d) $|P(\theta)|<\left(2+\frac{3}{\pi}\right) N+O(1)$ for all $\theta$.

Proof of Theorem 1. A straightforward calculation shows that for integers $m, r$ with $0 \leqslant m, r<N$ we have

$$
P\left(-\frac{m N+r}{N^{2}}\right)=N \exp \left(-2 \pi i \frac{m N+r}{N^{2}} r\right)
$$

and (a) follows.

To obtain (b) we define the functions

$$
G(\theta)=\frac{i N}{2 \pi}\left(1-z^{N}\right) \sum_{k=-\infty}^{\infty} \frac{z^{k N}}{k+N \theta}
$$

and

$$
H(\theta)=\frac{i N}{2 \pi}\left(1-z^{N}\right) \sum_{k=0}^{N-1} \frac{z^{k N}}{k+N \theta},
$$


and the error functions

$$
R(\theta)=P(\theta)-H(\theta) \text { and } S(\theta)=H(\theta)-G(\theta)
$$

To estimate $R(\theta)$ we let

$$
\phi(x)=\frac{\sin x}{2(1-\cos x)}-\frac{1}{x} \text { and } \psi(x)=\frac{1}{1-\exp (i x)}-\frac{i}{x}=\frac{1}{2}+i \phi(x) .
$$

We can now write

$$
R(\theta)=\left(1-z^{N}\right) \psi(2 \pi \theta)+\sum_{k=0}^{N-1} z^{(k+1) N}\left\{\psi\left(2 \pi\left(\frac{k+1}{N}+\theta\right)\right)-\psi\left(2 \pi\left(\frac{k}{N}+\theta\right)\right)\right\},
$$

which, together with the facts that $\phi^{\prime}(x)<0,-2 \pi<x<2 \pi$, and $-1+\varepsilon \leqslant \theta \leqslant-\varepsilon$, $\varepsilon<\frac{1}{2}$ yields

$$
\begin{aligned}
|R(\theta)| \leqslant & 2|\psi(2 \pi \theta)|+\sum_{k=0}^{N-1}\left|\psi\left(2 \pi\left(\frac{k+1}{N}+\theta\right)\right)-\psi\left(2 \pi\left(\frac{k}{N}+\theta\right)\right)\right| \\
& =2|\psi(2 \pi \theta)|+\sum_{k=0}^{N-1}\left\{\phi\left(2 \pi\left(\frac{k}{N}+\theta\right)\right)-\phi\left(2 \pi\left(\frac{k+1}{N}+\theta\right)\right)\right\} \\
& =2|\psi(2 \pi \theta)|+\frac{1}{2 \pi}\left(\frac{1}{1+\theta}-\frac{1}{\theta}\right) \\
& <2\left\{\frac{1}{2}+\phi(2 \pi(-1+\varepsilon))\right\}+\frac{1}{2 \pi \varepsilon(1-\varepsilon)} \\
& <1+\frac{2}{\pi}+\frac{2}{\pi \varepsilon} .
\end{aligned}
$$

To estimate $S(\theta)$, we observe that $1-N \theta \geqslant \varepsilon N>1$ and $N(1+\theta) \geqslant \varepsilon N>1$, so that we may apply Lemma 1 with $x= \pm N \theta$ and $T=\varepsilon N$ to get

$$
\begin{aligned}
|S(\theta)| & =\frac{N}{2 \pi}\left|1-z^{N}\right|\left|\sum_{k=-\infty}^{-1} \frac{z^{k N}}{k+N \theta}+\sum_{k=N}^{\infty} \frac{z^{k N}}{k+N \theta}\right| \\
& \leqslant \frac{N}{2 \pi}\left|1-e^{2 \pi i N \theta}\right|\left\{\left|\sum_{k=0}^{\infty} \frac{e^{-2 \pi i k N \theta}}{k+1-N \theta}\right|+\left|\sum_{k=0}^{\infty} \frac{e^{2 \pi i k N \theta}}{k+N(1+\theta)}\right|\right\}<\frac{3}{\pi \varepsilon} .
\end{aligned}
$$

Next we apply Lemma 2 with $x=r=N \theta$ to conclude that

$$
|G(\theta)|=N \text { for all } \theta
$$

Finally, combining (1), (2) and (3) with the fact that $P(\theta)=G(\theta)+R(\theta)+S(\theta)$, we obtain (b). 
For (c) we assume that $|\theta|<\frac{1}{4}$ and apply the techniques of the proof of (b) to get

$$
\begin{aligned}
P(\theta) & =\frac{i N}{2 \pi}\left(1-e^{2 \pi i N \theta}\right)\left(\sum_{k=0}^{\left[\frac{1}{2} N\right]} \frac{e^{2 \pi i k N \theta}}{k+N \theta}+\sum_{k=\left[\frac{1}{2} N\right]+1}^{N-1} \frac{e^{2 \pi i k N \theta}}{k-N+N \theta}\right)+O(1) \\
& =\frac{i N}{2 \pi}\left(1-e^{2 \pi i N \theta}\right)\left(\sum_{k=0}^{\infty} \frac{e^{2 \pi i k N \theta}}{k+N \theta}+e^{2 \pi i N^{2} \theta} \sum_{k=-\infty}^{-1} \frac{e^{2 \pi i k N \theta}}{k+N \theta}\right)+O(1)
\end{aligned}
$$

If $N$ is odd, (4) immediately implies that

$$
P\left(\frac{1}{2 N}\right)=O(1)
$$

For $N$ even, (4) yields

$$
\begin{aligned}
P\left(\frac{N-1}{2 N^{2}}\right)= & \frac{2 i N}{\pi}\left(\sum_{k=0}^{\infty} \frac{(-1)^{k} e^{-\pi i(k / N)}}{2 k+1}-e^{(\pi i / N)} \sum_{k=0}^{\infty} \frac{(-1)^{k} e^{\pi i(k / N)}}{2 k+1}\right)+O(1) \\
= & \frac{2 i N}{\pi}\left(1-e^{\pi i / N}\right) \sum_{k=0}^{\infty} \frac{(-1)^{k} \cos (k \pi / N)}{2 k+1}+\frac{4 N}{\pi} \sum_{k=1}^{\infty} \frac{(-1)^{k} \sin (k \pi / N)}{2 k+1} \\
& +O(1)=O(1),
\end{aligned}
$$

where the final estimate follows, for example, from Gradshteyn and Ryzhik [4], p. 38, formulas $1.442-3$ and 4 , and (c) is proven.

To establish (d) we may assume that $|\theta|<\frac{1}{4}$, and we let $N \theta=M+t$, where $M=[N \theta]$. For $M \leqslant-1$ we have, by Lemmas 1 and 2 ,

$$
\left|\left(1-e^{2 \pi i N \theta}\right) \sum_{k=0}^{\infty} \frac{e^{2 \pi i k N \theta}}{k+N \theta}\right|=\left|\left(1-e^{2 \pi i t}\right)\left(\sum_{k=-\infty}^{\infty} \frac{e^{2 \pi i k t}}{k+t}-\sum_{k=-\infty}^{M-1} \frac{e^{2 \pi i k t}}{k+t}\right)\right|<2 \pi+3 .
$$

It is equally trivial to obtain an identical estimate for $M \geqslant 0$, and the same method also yields

$$
\left|\left(1-e^{2 \pi i N \theta}\right) \sum_{k=-\infty}^{-1} \frac{e^{2 \pi i k N \theta}}{k+N \theta}\right|<2 \pi+3 \text { for }|\theta|<\frac{1}{4} .
$$

Finally, (d) follows from (4), (5), and (6), and the proof of Theorem 1 is complete.

We point out several immediate consequences of this theorem. First, if $\alpha$ is a fixed real number and if we define $Q$ by $Q(\theta)=P(\theta+\alpha)$, it is obvious that $Q \in \mathscr{G}_{N^{2}-1}$. Therefore, the bad interval in (b) can be shifted to any interval of length $2 \varepsilon$.

Second, if we are interested in a fixed subinterval of the unit circle, the estimate in (b) becomes quite remarkable. For example, setting $\varepsilon=\frac{1}{4}$ we obtain

Corollary 1. On the unit semicircle $-\frac{3}{4} \leqslant \theta \leqslant-\frac{1}{4}$ we have $|P(\theta)|=N+E$, where $|E|<1+22 \pi^{-1}<9$.

Third, by employing, for example, a result of Beller [1], we are able to extend Theorem 1 to the case of polynomials of arbitrary degree. We have 
Corollary 2. Let $n$ be a positive integer. Then there is a $g \in \mathscr{G}_{n}$ satisfying

(e) For any $\varepsilon,\left[n^{\frac{1}{2}}\right]^{-1}<\varepsilon<\frac{1}{2}, \quad|g(\theta)|=n^{\frac{1}{2}}+E$ for $-1+\varepsilon \leqslant \theta \leqslant-\varepsilon$, where $|E|<2+2 \pi^{-1}+5(\pi \varepsilon)^{-1}+2 n^{t}$, and

(f) $|g(\theta)|<(2+3 / \pi) n^{\frac{1}{2}}+2 n^{\frac{1}{4}}+O(1)$ for all $\theta$.

Proof of Corollary 2. Let $N=\left[n^{\frac{1}{2}}\right], m=n-N^{2}$, and choose $P(\theta)$ as in Theorem 1 . By Beller's result [1], we can choose $f \in \mathscr{G}_{m}$ such that $|f(\theta)|<1 \cdot 172 \mathrm{~m}^{\frac{1}{2}}<2 n^{\frac{1}{4}}$ for all $\theta$. If we now let $g(\theta)=P(\theta)+z^{N^{2}} f(\theta)$, the required estimates follow immediately from the Theorem, and Corollary 2 is proven.

Finally we observe that if we choose $\varepsilon$ in Corollary 2 to be, for example, $n^{-\frac{1}{2}} \log n$, we obtain the improvement of Littlewood's result mentioned in the introduction.

2. We now proceed with our construction of functions $G(\theta)$ which are almost in $\mathscr{G}_{n}$, and which satisfy $|G(\theta)|=n^{\frac{1}{2}}+O\left(n^{\frac{1}{2}}\right)$ for all $\theta$. Toward this end, we have

THEOREM 2. Let $n$ be a positive integer, and let $N$ be the even positive integer satisfying $N^{2} \leqslant n<(N+2)^{2}$. Then there exist functions $f$ and $g$ such that

(A) $z^{N^{2}} f+g \in \mathscr{G}_{n}$, and

(B) $|f(\theta)+g(\theta)|=n^{\frac{1}{2}}+O\left(n^{\frac{1}{2}}\right)$, where the error is uniform in $\theta$ and $n$.

Remark. It will be seen from the following construction that $g$ consists of two parts; a polynomial in $\mathscr{G}_{n-2 n^{3 / 4}+O\left(n^{1 / 2}\right)}$, plus $z$ to an integral power multiplied by a polynomial with coefficients of modulus $\frac{1}{2}$ and degree $4 n^{\frac{1}{4}}+O\left(n^{\frac{1}{2}}\right)$ in $z^{\frac{1}{2}}$. Also, $f$ is a function of precisely the same type as the second part of $g$, just described. Thus we see that, except for a relatively small number (i.e., $O\left(n^{\frac{z}{2}}\right)$ ) of terms, $f+g \in \mathscr{G}_{n}$, and so we can use $f+g$ as the function $G(\theta)$ mentioned above.

Proof of Theorem 2. Define $\delta$ by $\delta N=\left[N^{\frac{1}{2}}\right]$, let $z=\exp (2 \pi i \theta),-\frac{1}{2} \leqslant \theta \leqslant \frac{1}{2}$, let $m=n-N^{2}$, and choose $G_{1} \in \mathscr{G}_{m}$ such that $\left|G_{1}(\theta)\right|=O\left(n^{\frac{1}{2}}\right)$ (see the proof of Corollary 2). Define the functions

$$
\begin{aligned}
& f(\theta)=\frac{1}{2} z^{\left(\frac{1}{2} N-\delta N\right) N} \sum_{j=-\frac{1}{2} N-\delta N}^{-\frac{1}{2} N+\delta N-1} z^{j N} \sum_{k=0}^{2 N-1} \exp k \pi i\left(j N^{-1}+\theta\right) \\
& F(\theta)=z^{\left(\frac{1}{2} N-\delta N\right) N}\left\{\sum_{\frac{1}{2}}^{\frac{1}{2} N+\delta N-1} \sum_{j=\frac{1}{2} N-\delta N}^{j N N} z_{k=0}^{2 N-1} \exp k \pi i\left(j N^{-1}+\theta\right)\right. \\
& \left.+\sum_{j=-\frac{1}{2} N+\delta N}^{\frac{1}{2} N-\delta N-1} z^{j N} \sum_{k=0}^{N-1} \exp 2 k \pi i\left(j N^{-1}+\theta\right)\right\},
\end{aligned}
$$

and

$$
g(\theta)=F(\theta)+z^{N^{2}} G_{1}(\theta) .
$$


A straightforward calculation yields (A). To establish (B) we proceed as in the proof of Theorem 1(b), and we obtain

$$
\begin{aligned}
f(\theta)+g(\theta) & =z^{\left(\frac{1}{2} N-\delta N\right) N}\left(1-z^{N}\right) \frac{i}{2 \pi} \sum_{j=-\frac{1}{2} N-\delta N}^{\frac{1}{2} N+\delta N-1} \frac{z^{j N}}{(j / N)+\theta}+O\left(\delta^{-1}\right)+O\left(n^{\frac{1}{2}}\right) \\
& =z^{\left(\frac{1}{2} N-\delta N\right) N}\left(1-z^{N}\right) \frac{i N}{2 \pi} \sum_{j=-\infty}^{\infty} \frac{z^{j N}}{j+N \theta}+O\left(n^{\frac{1}{4}}\right) .
\end{aligned}
$$

Therefore, by Lemma $2,|f+g|=N+O\left(n^{\frac{1}{4}}\right)=n^{\frac{1}{2}}+O\left(n^{\frac{1}{5}}\right)$, and the proof of Theorem 2 is complete.

In conclusion we mention a result with a similar flavour to Theorem 2 by Beller and Newman [2], who prove the Littlewood conjecture, with $A_{1}$ and $A_{2}$ both much smaller than 1 , for polynomials whose coefficients are bounded by 1 in modulus.

\section{References}

1. E. Beller, "Polynomial Extremal Problems in $L^{p}$," Proc. Amer. Math. Soc., 30 (1971), 247-259.

2. 45 (1974), 463-465.

3. Paul Erdôs, "Some Unsolved Problems", Michigan Math. J., 4 (1957), 291-300.

4. I. S. Gradshteyn, and I. M. Ryzhik, Table of Integrals, Series, and Products (New York, Academic

5. J. E. Littlewood, "On Polynomials $\sum_{0}^{n} \pm z^{m}, \sum_{0}^{n} e^{\alpha_{m}{ }^{\prime}} z^{m}, z=e^{i \theta}$ ", J. London Math. Soc., 41
(1966), 367-376. (1966), 367-376.

6. G. G. Lorentz, Appraximation of Functions (New York, Holt, Rinehart and Winston, 1966).

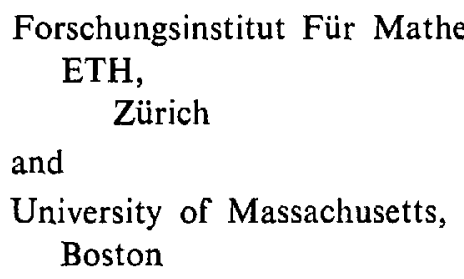

